University of Northern lowa

UNI ScholarWorks

Faculty Publications

Faculty Work

$6-2002$

\title{
Electron-Hole Coupling and the Charge Density Wave Transition inTiSe2
}

T. E. Kidd

University of Illinois at Urbana-Champaign

T. Miller

University of Illinois at Urbana-Champaign

See next page for additional authors

Let us know how access to this document benefits you

Copyright (C2002 T.E. Kidd, T. Miller, M.Y. Chou, and T.-C. Chiang. The copyright holder has granted permission for posting.

Follow this and additional works at: https://scholarworks.uni.edu/phy_facpub

Part of the Physics Commons

\section{Recommended Citation}

Kidd, T. E.; Miller, T.; Chou, M. Y.; and Chiang, T.-C., "Electron-Hole Coupling and the Charge Density Wave Transition inTiSe2" (2002). Faculty Publications. 17.

https://scholarworks.uni.edu/phy_facpub/17

This Article is brought to you for free and open access by the Faculty Work at UNI ScholarWorks. It has been accepted for inclusion in Faculty Publications by an authorized administrator of UNI ScholarWorks. For more information, please contact scholarworks@uni.edu. 
Authors

T. E. Kidd, T. Miller, M. Y. Chou, and T.-C. Chiang 


\title{
Electron-Hole Coupling and the Charge Density Wave Transition in $\mathrm{TiSe}_{2}$
}

\author{
T. E. Kidd, ${ }^{1}$ T. Miller, ${ }^{1}$ M. Y. Chou, ${ }^{2}$ and T.-C. Chiang ${ }^{1, *}$ \\ ${ }^{1}$ Department of Physics, University of Illinois at Urbana-Champaign, 1110 West Green Street, \\ Urbana, Illinois 61801-3080 \\ and Frederick Seitz Materials Research Laboratory, University of Illinois at Urbana-Champaign, \\ 104 South Goodwin Avenue, Urbana, Illinois 61801-2902 \\ ${ }^{2}$ School of Physics, Georgia Institute of Technology, Atlanta, Georgia 30332-0430
}

(Received 8 November 2001; published 17 May 2002)

\begin{abstract}
Angle-resolved photoemission is employed to measure the band structure of $\mathrm{TiSe}_{2}$ in order to clarify the nature of the $(2 \times 2 \times 2)$ charge density wave transition. The results show a very small indirect gap in the normal phase transforming into a larger indirect gap at a different location in the Brillouin zone. Fermi surface topology is irrelevant in this case. Instead, electron-hole coupling together with a novel indirect Jahn-Teller effect drives the transition.
\end{abstract}

DOI: $10.1103 /$ PhysRevLett.88.226402

$\mathrm{TiSe}_{2}$ is one of the simplest and most studied charge density wave $(\mathrm{CDW})$ compounds. It undergoes a $(2 \times 2 \times 2)$ transition below $T_{c}=200 \mathrm{~K}$ due to the freezing of a zone boundary phonon mode [1-3]. Surprisingly, the mechanism for this seemingly elementary transition remains a puzzle after decades of research. It does not seem to fit the standard textbook model based on Fermi surface nesting [4]. Self-consistent calculations of the electronic structure have found the normal phase to be metallic with a small negative indirect gap across $\Gamma$ and $L$, and there is no obvious nesting $[5,6]$. Alternate theoretical ideas have been proposed, including a band Jahn-Teller effect $[7,8]$ and a carrier-suppressed ferroelectric transition [9], but none of these has been verified by experiment.

This issue can be resolved, in principle, by angleresolved photoemission measurements of the band structure [10-15]. Numerous attempts, however, have yielded inconsistent results, and the data are generally too coarse or sparse to allow a detailed understanding of the effect of the transition on the band structure. The present work reexamines this system with a high resolution. Contrary to theoretical findings of a band overlap in the normal phase, our results show a very small indirect gap across $\Gamma$ and $L$. Upon cooling to below $T_{c}$, the occupied valence band is depressed in energy, resulting in a larger gap and a lowering of the electronic energy. The minimum of the unoccupied conduction band moves to a new location, and the gap remains indirect. No Fermi surface nesting is involved in this semiconductor-to-semiconductor transition, and none of the previously proposed models can properly describe the behavior. Our analysis in terms of a reduced Hamiltonian indicates that electron-hole coupling and an indirect Jahn-Teller effect drive this unconventional CDW transition.

The photoemission experiment was performed at the Synchrotron Radiation Center in Stoughton, Wisconsin. The system energy resolution was set to less than $20 \mathrm{meV}$, and the angular resolution was $0.35^{\circ}$. The samples were grown by iodine vapor transport with a temperature gra-
PACS numbers: 71.45.Lr, 71.20.Nr, 71.30.+h, 79.60.Bm

dient of $570-640{ }^{\circ} \mathrm{C}$. Sample stoichiometry was checked with resistivity and $\mathrm{x}$-ray measurements [3].

Figure 1(a) shows a drawing of the Brillouin zone in the normal phase. Below $T_{c}$, a $(2 \times 2 \times 2)$ distortion folds the $L$ point back to the zone center at $\Gamma$. The discussion below uses the $k$ space notation for the normal phase, and the peak assignment is based on the calculated band structure. Our detailed survey of the bands shows that $\mathrm{TiSe}_{2}$ in the normal phase has a small, almost zero, indirect gap with the occupied valence band maximum at $\Gamma$ and the unoccupied conduction band minimum at $L$. Figure 1(b) shows spectra taken near the $\Gamma$ point at two temperatures, one above and the other below $T_{c}$. The gray scale indicates the photoemission intensity, and the dispersion direction (horizontal axis) is along the $\Gamma-K$ direction. At $260 \mathrm{~K}$, two spin-orbit-split [11] valence bands derived from the Se $4 p$ states are seen to follow closely a parabolic dispersion. The top of the upper valence band lies very close to the Fermi level. Fitting of the energy distribution curves at various $k$ with a Fermi-Dirac function for thermal cutoff has revealed the top of the upper valence band to be at a binding energy of $0.030 \mathrm{eV}$. This small binding energy leads to a significant emission intensity at the Fermi level, and the band is thermally populated by holes. This emission at the Fermi level is not due to a hole pocket at $\Gamma$ as reported in some previous studies. Upon cooling to $100 \mathrm{~K}$, the band structure near $\Gamma$ is significantly altered. The top of the upper band is depressed to higher binding energies, and the lower band is similarly affected, although to a lesser degree. Both bands become considerably flattened near the zone center and are no longer parabolic.

Figure 1(c) shows spectra taken as before but centered at the $L$ point [16]. The dispersion direction (horizontal axis) is along the $L-H$ direction. Above $T_{c}$, the main emission is almost right at the Fermi level, with a broad tail toward higher binding energies. A linear cut through $L$ yields the spectrum shown in Fig. 2(a). The Fermi level cuts through the main peak above the half-height point (in fact, almost at the peak), indicating that the corresponding state lies 
(a)

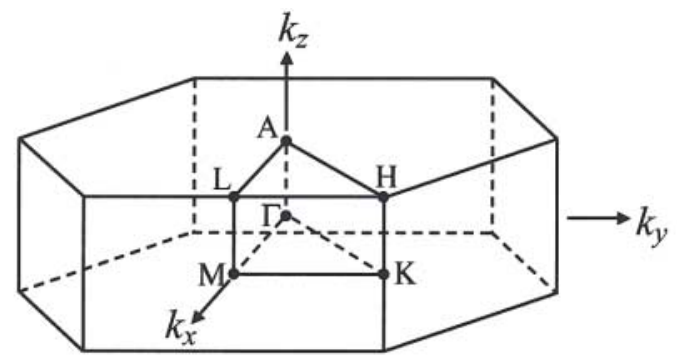

(b)

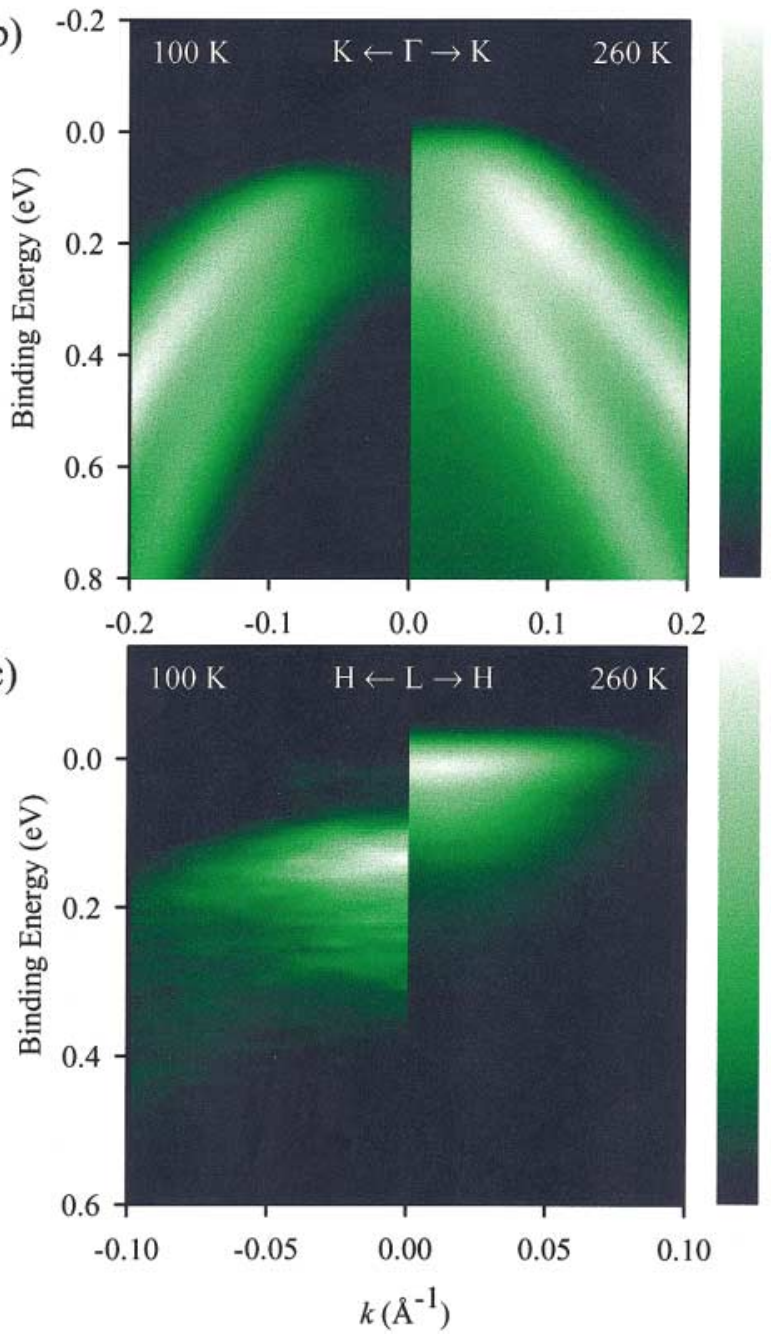

FIG. 1 (color). (a) Brillouin zone of $\mathrm{TiSe}_{2}$ in the normal phase. (b) Photoemission spectra taken near $\Gamma$ at $100 \mathrm{~K}$ (left) and $260 \mathrm{~K}$ (right) with a photon energy of $13 \mathrm{eV}$. The dispersion direction (horizontal axis) is along $\Gamma-K$, with zero at $\Gamma$. (c) Photoemission spectra taken near $L$ at $100 \mathrm{~K}$ (left) and $260 \mathrm{~K}$ (right) with a photon energy of $18 \mathrm{eV}$. The dispersion direction (horizontal axis) is along $L-H$, with zero at $L$.

just above the Fermi level. The main peak seen here is the tail of an otherwise unoccupied conduction band state derived from the Ti $3 d$ level. This is not, as reported in some previous studies, emission from an occupied electron pocket. At 100 K, Figs. 1(c) and 2(b) reveal a small Fermi edge at $L$ derived from the same Ti level, now moved up in energy and having a much smaller thermal tail in the spectrum. The spectrum is instead dominated by two peaks

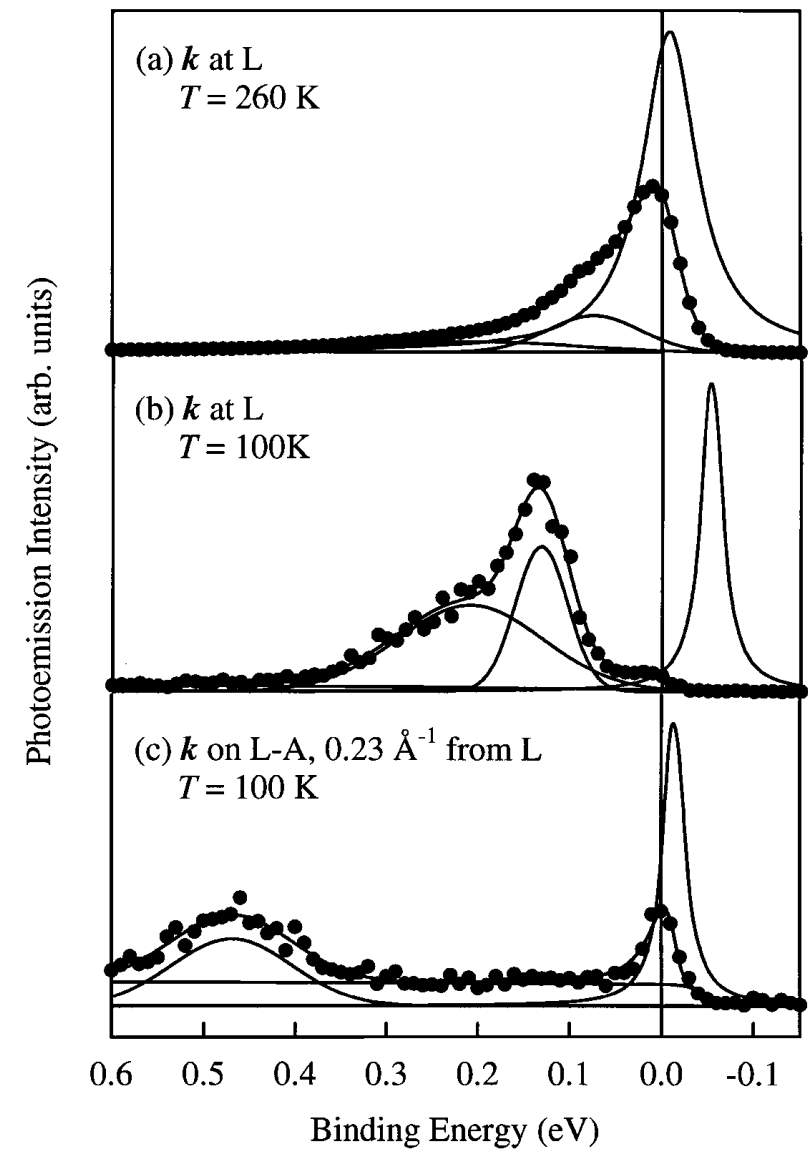

FIG. 2. Energy distribution curves taken at indicated points in $k$ space and at indicated temperatures. The decomposition of the curves into peaks and a Shirley background function is indicated.

at about 0.13 and $0.21 \mathrm{eV}$. These can be identified as umklapp features derived from the Se bands at $\Gamma$ folded onto $L$ due to the $(2 \times 2 \times 2)$ lattice distortion. Similar peaks, with much reduced intensities, can be seen in Fig. 2(a), which can be attributed to fluctuation, or dynamic umklapp effects associated with the Kohn anomaly at $L$ for $T>T_{c}$ [17].

To further elucidate the band structure near the conduction band minimum, data were taken near the $L$ point along the $L-A$ line. Figure 2(c) shows such a spectrum at $100 \mathrm{~K}$. As expected from band calculations, the folded Se bands have shifted to higher binding energies. The Ti conduction band at this point is just slightly above the Fermi level and gives rise to a peak almost right on the Fermi level. This spectrum illustrates that the conduction band minimum in the CDW phase deviates significantly from its initial position at $L$.

The experimental band structure is summarized in the composite band diagrams in Fig. 3. Included in each diagram are the valence bands below the Fermi level (Se $4 p$ ) near $\Gamma$ and the conduction bands above the Fermi level (Ti $3 d$ ) near $L$. There is a considerable anisotropy for the bands near $L$, and the diamonds and open circles are data points along the $L-H$ and $L-A$ principal symmetry 


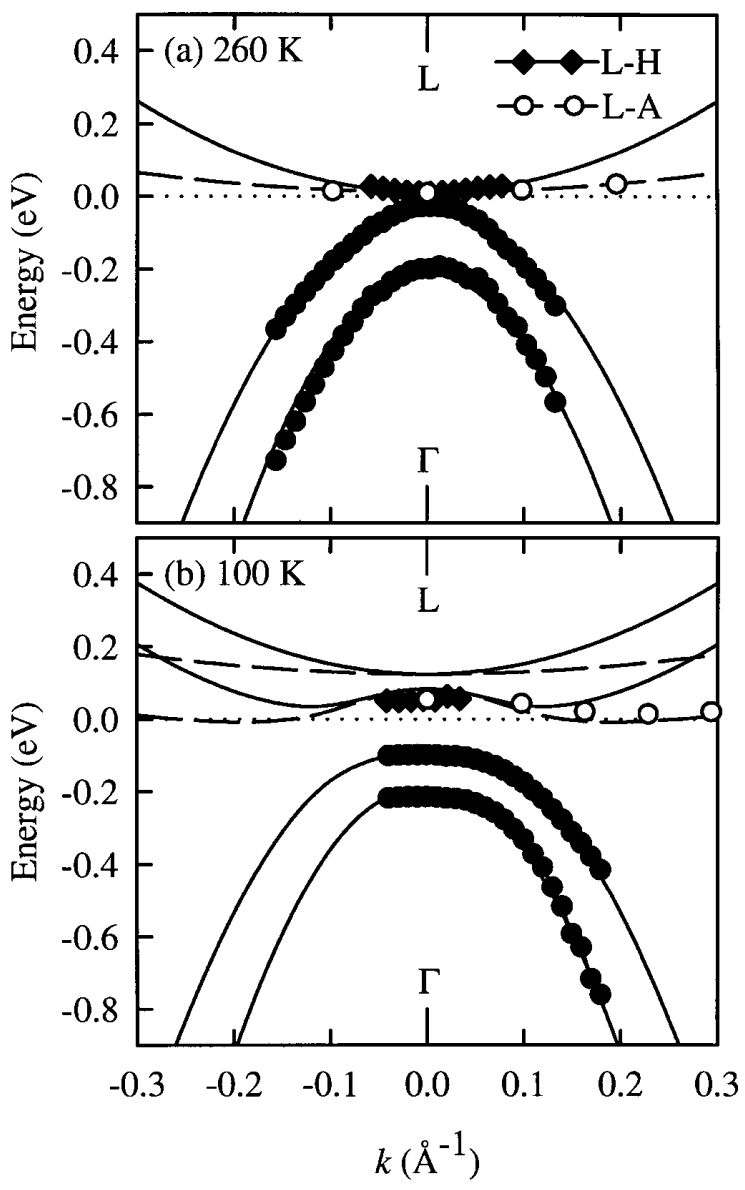

FIG. 3. (a) Band structure of $\mathrm{TiSe}_{2}$ in the normal phase at $260 \mathrm{~K}$. The symbols represent data and the curves are fits. (b) Same as (a) except $T=100 \mathrm{~K}$. The dispersion direction for the valence bands (filled circles and solid curves) is along $\Gamma-K$ (highly isotropic near $\Gamma$ ), with zero at $\Gamma$. The dispersion directions for the conduction bands are along $L-H$ (solid curves and solid diamonds) and $L-A$ (dashed curves and open circles), with zero at $L$.

directions, respectively. The curves in Fig. 3 are best fits of the experimental dispersion curves based on the reduced Hamiltonian:

$$
H=\left(\begin{array}{ccccc}
C & D & D & I & I^{\prime} \\
D & C & D & I & I^{\prime} \\
D & D & C & I & I^{\prime} \\
I & I & I & V & 0 \\
I^{\prime} & I^{\prime} & I^{\prime} & 0 & V^{\prime}
\end{array}\right)+\Delta E_{F},
$$

which describes the essential physics within the subspace near the gap. The diagonal matrix elements $V$ and $V^{\prime}$ represent the two occupied spin-orbit-split Se $4 p$ valence bands in the normal phase, and are paraboloids of the form

$$
E_{i}+\frac{\hbar^{2}\left(k_{x}^{2}+k_{y}^{2}\right)}{2 m_{i}},
$$

where $i=V$ or $V^{\prime}, E_{V}$ and $E_{V^{\prime}}$ are the band edge energies, and $m_{V}$ and $m_{V^{\prime}}$ are the effective masses. The diagonal element $C$ in Eq. (1) represents the unoccupied $\mathrm{Ti}$ $3 d$ conduction band in the normal phase. It is also a pa- raboloid, but is centered about the $L$ point and has different effective masses along the two principal symmetry directions $L-A$ and $L-H$. It appears three times in $H$ due to the threefold degeneracy of the conduction band, as there are three independent $L$ points in the first Brillouin zone. The diagonal elements $C, V$, and $V^{\prime}$ alone (with $I=I^{\prime}=D=\Delta E_{F}=0$ ) give rise to the parabolic bands shown in Fig. 3(a) for the normal phase.

The off-diagonal constants $I, I^{\prime}$, and $D$ are interactions that drive the system into the CDW phase. $I$ and $I^{\prime}$ represent coupling between the valence and conduction bands. $D$ represents coupling among the three symmetryrelated conduction bands, and is sometimes referred to as a crystal-field-splitting term. The effect of $D$ alone is to split the three conduction bands into a lower, nondegenerate $A$ band and a higher, doubly degenerate $E$ band. The $E$ band is unoccupied and not observed in the experiment. The $A$ band, pushed down in energy due to the interaction, would overlap with the top valence band $V$. This overlap is, however, avoided by the coupling term $I$. As a result of this interaction, the parabolic valence bands in the normal phase become flat topped as seen in Fig. 3(b). The $A$ band now exhibits split minima in $k$ as seen in the experiment, and the conduction band minimum is no longer at the $L$ point.

We have carried out first-principles density-functional calculations of the band structure. The normal phase is found to have a small negative gap, in agreement with previous calculations $[5,6]$, but in disagreement with the experiment. Furthermore, the total energy of the normal phase and the CDW phase is essentially the same with no obvious energy lowering associated with the lattice distortion. Both findings are not surprising for such a small-gap system, and suggest that not all many-body effects are captured in the local-density approximation.

All evidence thus points to electron-hole coupling (or an excitonic mechanism) as an important ingredient for the transition. This coupling was first discussed by Knox [18] and later developed further by Kohn [19]. The Hamiltonian in Eq. (1) is similar to the one used by Kohn, with $I$ and $I^{\prime}$ representing the electron-hole coupling. The only difference is that the conduction band degeneracy and the spin-orbit splitting of the valence bands are included in our analysis. The key feature or requirement of this mechanism is a small gap accentuating the electron-hole coupling. In the present case, the lattice distortion leads to a splitting of the degenerate conduction bands. This is similar to the Jahn-Teller effect except that the splitting occurs in the unoccupied states, and there is no energy lowering for the system. The bottom conduction band, however, cannot overlap the valence band due to the electron-hole coupling, and the consequence is that the top of the valence band gets pushed down, resulting in a lowering of the system energy. We call this an indirect Jahn-Teller effect, as the occupied band itself does not split, but is instead pushed down by the splitting of the conduction bands. The 
TABLE I. Parameters for the Hamiltonian. All energies are in $\mathrm{eV}$, and the effective masses are expressed in terms of the free electron mass.

\begin{tabular}{lccc}
\hline \hline & Ti band & Se band 1 & Se band 2 \\
\hline Band edge & 0.014 & -0.035 & -0.215 \\
Effective mass & $2.80(L-H) ;$ & -0.29 & -0.21 \\
& $6.42(L-A)$ & & \\
\hline$I$ & $I^{\prime}$ & $D$ & $\Delta E_{F}$ \\
0.048 & 0.026 & 0.051 & -0.044 \\
\hline
\end{tabular}

conduction band degeneracy and splitting, ignored in the work of Kohn [18], turns out to be a critical feature in this case.

The parameters deduced from the band fitting are listed in Table I. The effective masses for the bands and the splitting of the conduction bands are similar to the results of our density functional calculations. The magnitudes of $I$ and $I^{\prime}$ are small and consistent with the usual excitonic coupling energy found in semiconductors. The term $\Delta E_{F}$ represents an overall shift of the bands relative the Fermi level after the CDW transition. The exact location of the Fermi level in the gap can be affected by stoichiometry, doping, temperature, and possibly surface effects [12]. Measurements have shown that electrical transport in bulk $\mathrm{TiSe}_{2}$ for $T>T_{c}$ is dominated by holes [3]. Our experiment reveals significant hole and electron concentrations due to the small gap. Estimates based on our band structure agree that the hole channel dominates. For $T<T_{c}$, electron conduction dominates based on transport measurements [3]. This is consistent with the result shown in Fig. 3 that the conduction band minimum is very close to the Fermi level. It should be noted that even a perfectly cleaved surface can induce a perturbation in the region probed by photoemission. Although the effect is likely to be small for layered compounds, the results discussed above must be understood within this context.

To conclude, photoemission measurements of the band structure of $\mathrm{TiSe}_{2}$ reveal an unconventional CDW transition from a very small gap semiconductor in the normal phase to another semiconductor with a larger gap in the CDW phase. The gap remains indirect, albeit at different locations in the Brillouin zone. Previously reported electron and hole pockets do not exist in this system, and the emission at the Fermi level is due to thermal tails of bands that nearly graze the Fermi level. Since the system is never truly in a metallic state, concepts based on Fermi surface topology are irrelevant in this case. Our analysis clarifies the nature of the $\mathrm{CDW}$ transition as one involving a combination of electron-hole coupling and an indirect Jahn-Teller effect.

This work was supported by the U.S. Department of Energy under Grants No. DEFG02-91ER45439 (T. C. C.) and No. DEFG02-97ER45632 (M. Y. C.). The Synchrotron Radiation Center is supported by the U.S. National Science Foundation under Grant No. DMR-00-84402. An acknowledgment is made to the Donors of the Petroleum Research Fund, administered by the American Chemical Society, and to the U.S. National Science Foundation Grants No. DMR-99-75182 and No. 99-75470 for partial support of the synchrotron beam line operation.

*Email address: chiang@mrl.uiuc.edu

[1] J. A. Wilson and A. D. Yoffe, Adv. Phys. 18, 193 (1969).

[2] J. A. Wilson, F. J. Di Salvo, and S. Mahajan, Adv. Phys. 24, 117 (1975).

[3] F. J. Di Salvo, D. E. Moncton, and J. V. Waszczak, Phys. Rev. B 14, 4321 (1976).

[4] Charge Density Waves in Solids, edited by L. P. Gor'kov and G. Grüner (North-Holland, New York, 1989).

[5] A. Zunger and A. J. Freeman, Phys. Rev. B 17, 1839 (1978).

[6] G. A. Benesh, A. M. Woolley, and C. Umrigar, J. Phys. C 18, 1595 (1985).

[7] H. P. Hughes, J. Phys. C 10, L319 (1977).

[8] Structural Phase Transitions in Layered Transition Metal Compounds, edited by K. Motizuki (Reidel, Boston, 1986).

[9] R. M. White and G. Lucovsky, Nuovo Cimento Soc. Ital. Fis. 38B, 280 (1977).

[10] M. M. Traum, G. Margaritondo, N. V. Smith, J. E. Rowe, and F. J. Di Salvo, Phys. Rev. B 17, 1836 (1978).

[11] O. Anderson, R. Manzke, and M. Skibowski, Phys. Rev. Lett. 55, 2188 (1985).

[12] G. Karschnick, O. Anderson, W. Drube, and M. Skibowski, Surf. Sci. 155, 46 (1985).

[13] N. G. Stoffel, S. D. Kevan, and N. V. Smith, Phys. Rev. B 31, 8049 (1985).

[14] E. Pehlke, W. Schattke, O. Anderson, R. Manzke, and M. Skibowski, Phys. Rev. B 41, 2982 (1990).

[15] Th. Pillo, J. Haez, H. Berger, F. Lévy, L. Schlapbach, and P. Aebi, Phys. Rev. B 61, 16213 (2000).

[16] We used a photon energy of $18 \mathrm{eV}$ to probe the $L$ point, determined by observing the turning point associated with the back folded Se band. This is consistent with Ref. [13].

[17] Phonon softening (Kohn anomaly) occurs above $T_{c}$, resulting in a peak in the scattering factor that can cause umklapp between $\Gamma$ and $L$. See M. Holt, P. Zschack, H. Hong, M. Y. Chou, and T.-C. Chiang, Phys. Rev. Lett. 86, 3799 (2001).

[18] R. S. Knox, Solid State Phys. Suppl. 5, 100 (1963).

[19] W. Kohn, Phys. Rev. Lett. 19, 439 (1967). 